\title{
Symposium THD : un traitement sans risque et sans douleur de la maladie hémorroïdaire ?
}

\author{
(C) Springer-Verlag France 2010
}

25

\section{Ligature élective des artères hémorroïdales sous contrôle Doppler avec mucopexie vs Anopexie circulaire selon Longo : Enjeux d'une étude prospective comparative Hemorroidal artery ligation with mucopexy vs Longo's procedure: \\ Challenge of a prospective comparative study}

P.-A. Lehur, G. Meurette

Clinique de chirurgie digestive et endocrine, Nantes

Mots clés : Maladie hémorroïdaire • Ligature des artères hémorroïdales • Anopexie circulaire agrafée

Résumé : Le traitement chirurgical de la maladie hémorroïdaire repose sur des techniques conventionnelles (hémorrö̈dectomie sous muqueuse) efficaces mais exposant à des risques significatifs de complications (manifestations douloureuses, hémorragie, sténose, incontinence) bien documentées dans la littérature. Des traitements alternatifs ont été proposés pour limiter les effets secondaires de ces interventions. L'anopexie circulaire selon Longo a ainsi prouvé son efficacité. De façon plus récente, la ligature élective des artères hémorroïdales sous contrôle Doppler a également été proposée. Il n'existe pas actuellement de données comparant les 2 techniques. De façon à déterminer les indications respectives, et évaluer l'efficacité des techniques récentes de traitement de la maladie hémorroïdaire, une étude prospective randomisée prospective est mise en place. Son objectif est de comparer les 2 approches en terme de récidive, ainsi qu'une description précise des effets secondaires à court et long terme de chacune des approches. La mise en place multicentrique de cet essai laisse à l'opérateur le choix du matériel utilisé pour chacune des deux techniques.
26

Bilan d'une année de désartérialisation de doppler guidée avec THD A year of dearterialization doppler guided with THD

M. Queralto, JP Chotard, G Bonnaud

Clinique des Cèdres F-31700 Cornebarrieu, France

Mail: michel.queralto@wanadoo.fr

Nous sommes trois proctologues non exclusifs (gastro-entérologues) et avons réalisé un peu plus 100 procédures en un an.

Nous avons connu plusieurs phases :

- une formation dispensée par le Professeur Paul Antoine LEHUR à partir de 7 patients tous stades confondus. Les résultats étaient très bons : correction du prolapsus, peu de douleurs, hospitalisation d'une nuit en post opératoire, aucune complication. «THD is simply the best».

- lors d'une deuxième phase deux attitudes étaient opposées :

- passionnée : «On peut tout faire si la mucopexie descend plus bas, si la mucopexie est plus importante en circonférence ». Avec ces modifications techniques nous avons observé des complications : 1 fissure postérieure (ischémie ?), une rectorragie importante (chute de 2 points d'hémoglobine) mais qui n'a nécessité ni transfusion, ni reprise chirurgicale, surtout deux thromboses circonférentielles. Si la première a bien réagi au traitement médical, la seconde à nécessité une intervention chirurgicale. Des récidives ultra précoces sont observées pour les hauts grades.

- raisonnée : Nous discutons des desiderata de chaque patient en visionnant une vidéo obtenue par un défécoscope. Il s'agit d'une cuvette WC équipée d'une caméra. Ce système permet d'évaluer l'importance du prolapsus, dans des conditions physiologiques de défécation, et en respectant l'intimité du patient. Les résultats obtenus lors de la défécoscopie sont tout à fait superposables aux anomalies constatées en per opératoires, le choix de la technique fait en pré opératoire est donc toujours respecté. Dans ces conditions, tous les stades peuvent être pris en charge, les patients sont satisfaits des résultats, nous n'avons pas de recul suffisamment important pour évaluer les récidives.

Résultats : nous n'avons observé aucune complication, dans $20 \%$ des cas il existe des faux besoins qui au maximum perdurent 10 jours. Le traitement pourrait être réalisé en ambulatoire mais le GHS de $300 €$ environ ne couvre pas l'achat du kit.

Actuellement l'attitude raisonnée est adoptée par les 3 opérateurs. Conclusion : la désartérialisation de doppler guidée est une intervention mini invasive, sans excision ce qui explique l'absence de complication notable et des suite opératoires peu douloureuses. L'efficacité semble bonne à court terme. 